(C) Elsevier Sequoia S.A., Lausanne - Printed in the Netherlands

\title{
CAVITATION DAMAGE PREDICTION
}

\author{
F. G. HAMMITT and M. K. DE
}

Department of Mechanical Engineering, Cavitation and Multiphase Flow Laboratory, University of Michigan, Ann Arbor, Michigan (U.S.A.)

(Received May 16, 1978)

\section{Summary}

New results from cavitating venturi water tests were used to reinforce the concept of cavitation erosion efficiency previously developed from tests in a vibratory facility with both water and sodium. The concept emerges from a technique which allows a priori prediction of eventual cavitation erosion rates in flow machines. Bubble collapse pulse height spectra obtained from submerged microprobes are correlated with measured erosion rates in given laboratory and/or field devices to allow this prediction. Preliminary results from such correlations are presented together with other measurements of the effects of gas content, velocity and cavitation condition upon the mechanical cavitation intensity as measured by the pulse height spectra.

New results from vibratory facility tests in tap water and synthetic seawater upon three materials of variable corrodability (304 stainless steel, 1018 carbon steel and 1100-0 aluminum) are presented. The ratio between maximum erosion rates for the saltwater and freshwater tests were found to increase toward unity as the mechanical cavitation intensity is increased, i.e. increased mean depth to penetration (MDPR), as expected on theoretical grounds.

The relation between the incubation period and $M D P R_{\max }$ was examined from the vibratory test results, and was found to depend upon the material properties as well as the fluid flow conditions.

\section{Introduction}

One of the major difficulties facing designers of liquid flow machinery where cavitation is a possibility is the present almost complete inability to predict eventual cavitation damage rates, or even their probable existence, from reasonably practical laboratory tests. Hence very expensive long term cavitation damage tests in near prototype scale conditions are often necessary. The primary purpose of the on-going research at the University of 
Michigan is to provide new data and techniques from laboratory scale work to help to bridge this gap in predicting capability.

It is hoped to attain this goal by using relatively more sophisticated acoustic techniques than have been generally applied to this problem in the past. The relation between general cavitation noise and erosion has recently been studied in several laboratories [1 - 7] for the purpose of predicting eventual damage rates. While no very general success has been attained, a relatively good relation between noise level and damage rate for specific units has been observed. It is hoped to improve the general utility of acoustic measurements for damage prediction by a more pertinent and sophisticated analysis of the acoustic data. Rather than using total noise amplitude or the amplitude within a fixed frequency band [1 - 4], the noise was analyzed in terms of a spectrum comprising the number of pressure pulses and their individual amplitudes. The initial results used a relatively standard cavitation damage vibratory facility $[8,9]$, and produced quite good correlations between spectral areas and the measured damage rate (mean depth of penetration rate (MDPR) based on surface area) as shown in Fig. 1 . These results were presented $[8,9]$ in terms of the cavitation erosion efficiency (Fig. 2) which is defined as the ratio between the measured acoustic power in the cavitation field and the erosion power. Erosion power is defined as the product of volume loss rate and material failure energy, considered here to be the ultimate resilience. This cavitation erosion efficiency (Fig. 2) was found to be numerically very small, as would be intuitively expected, but it remained relatively constant* over a broad range of test temperature and pressure [ 8 , 9] which provides further evidence for the probable validity of the concept. Figure $2[8,9]$ is typical.

Similar instrumentation installed in flow machines would allow an a priori prediction of the eventual damage rates to be encountered in such machines. Such a prediction is not otherwise possible in the present state of the art. Similar preliminary results have also been reported from Japan $[5,6]$ and Russia [7] .

The present paper provides related preliminary results from a water venturi system, as opposed to the previous results from a water and sodium vibratory facility $[8,9]$. It also presents new vibratory results which were designed to separate mechanical and corrosive cavitation erosion by using tests in freshwater and saltwater with materials of differing degrees of corrosivity tested over a range of mechanical intensities. Presumably the pulse spectra measure such mechanical intensities but are insensitive to corrosive intensities.

*That is within an order of magnitude, which is itself surprising considering the very gross assumptions involved. Figure 2 is not meant to show a meaningful correlation with temperature, but merely indicates the order of magnitude of cavitation efficiency and that it does not change with temperature. 


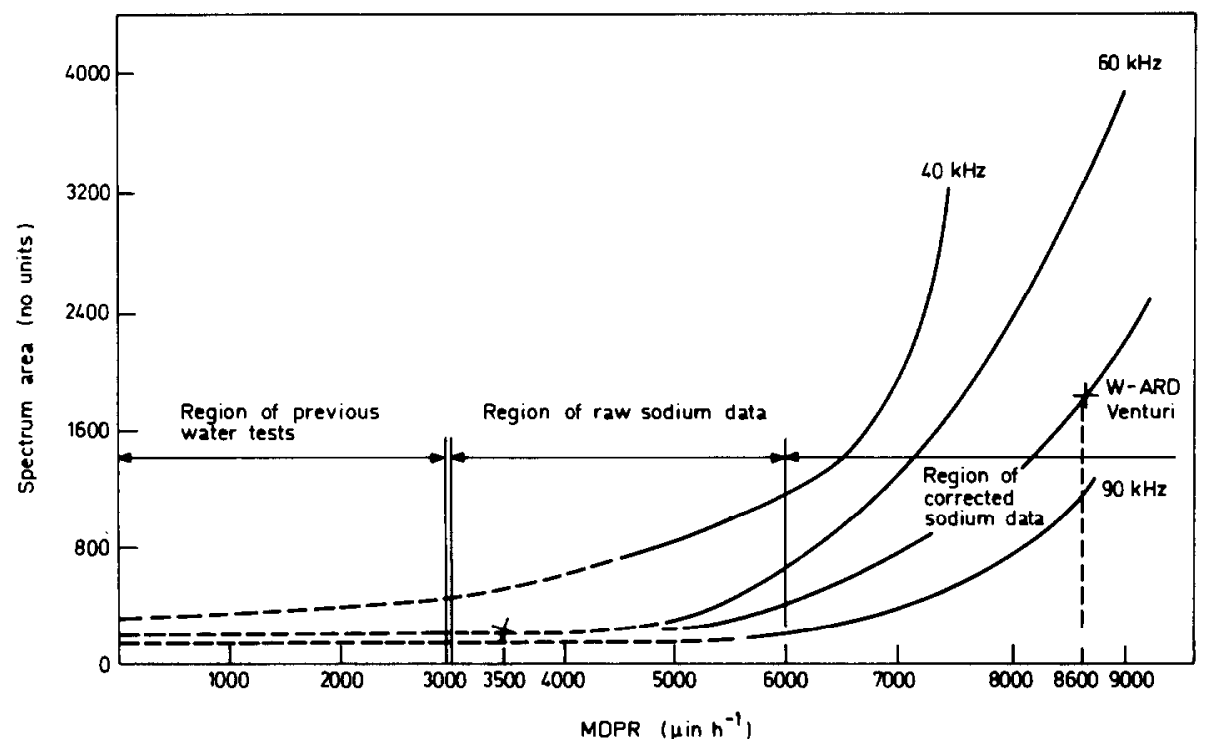

Fig. 1. Correlation of the pulse pressure spectrum area and MDPR.

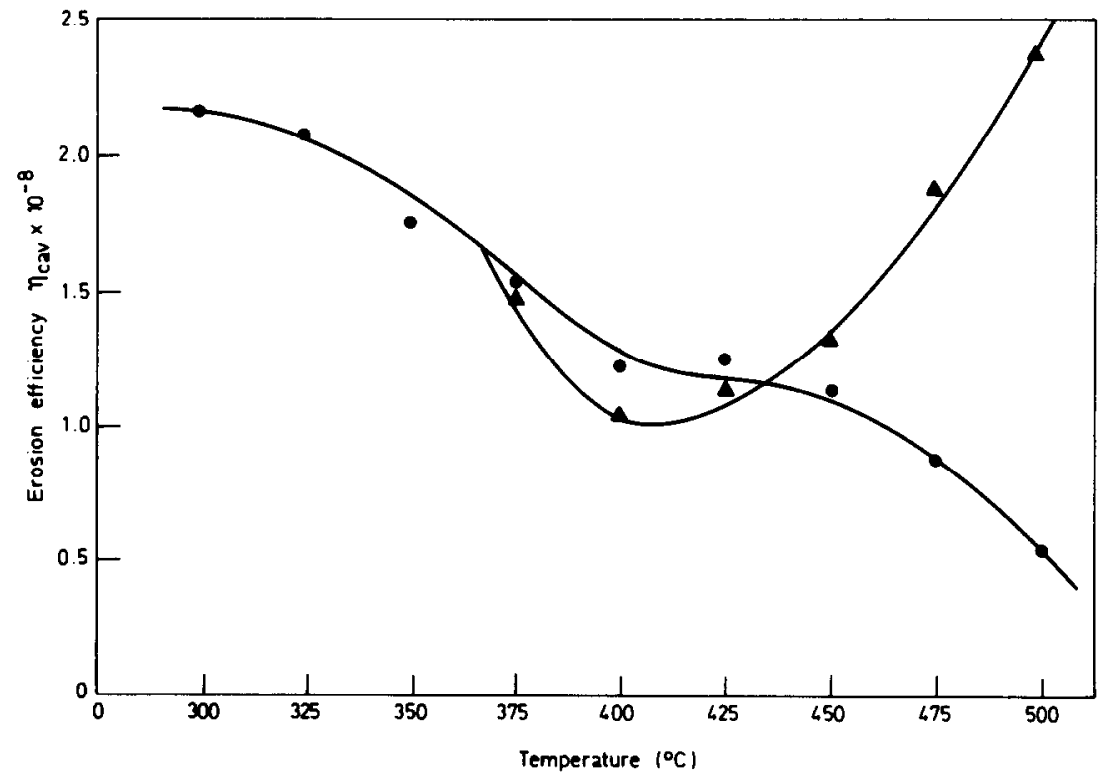

Fig. 2. Temperature $v s$. cavitation erosion efficiency in sodium for the vibratory facility $(70 \mathrm{kHz}$ cut-off frequency): $\Delta 2 \mathrm{~atm} ; \bullet 3 \mathrm{~atm}$.

\section{Saltwater and freshwater vibratory tests [10]}

Three materials of varying corrodability were tested in synthetic seawater $(2.4 \mathrm{wt} . \% \mathrm{NaCl})$ and in Ann Arbor tap water. These were 1100-0 alumi- 


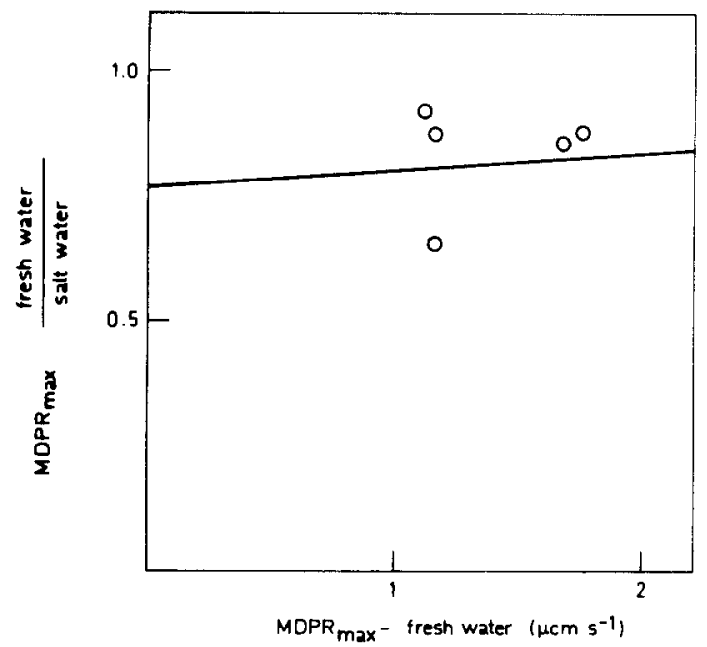

Fig. 3. Ratio of $M D P R_{\max }$ in freshwater and saltwater vs. MDPR $\max$ for freshwater (stainless steel 304 ): slope, $0.035 ; y$ intercept, 0.779 ; correlation, 0.0967 .

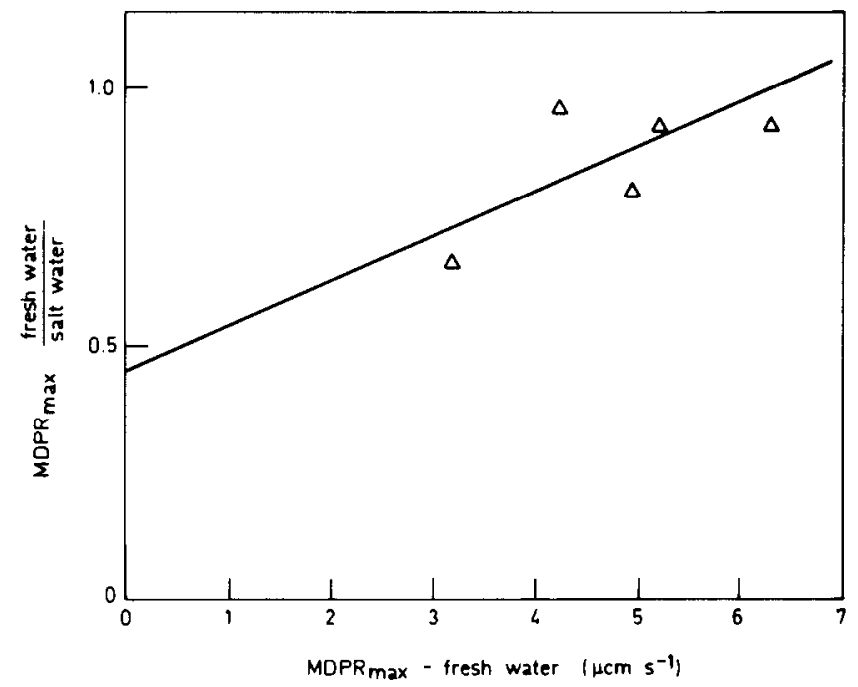

Fig. 4. Ratio of MDPR $_{\max }$ in freshwater and saltwater us. MDPR $_{\max }$ for freshwater (1018 carbon steel): slope, $0.084 ; y$ intercept, 0.455 ; correlation coefficient, 0.7255 .

num, 304 stainless steel and 1018 (cold-rolled) carbon steel. The mechanical cavitation intensity was varied for these tests by varying either the suppression pressure* or the temperature of the liquid. Suppression pressures of 2 and 3 bar were provided by compressed air cover gas, and the temperature of the liquid was varied from 20 to $105{ }^{\circ} \mathrm{C}$. The peak-to-peak horn ampli-

*Suppression pressure $=p-p_{\mathrm{v}}=$ NPSH $\times p$ where $p_{\mathrm{v}}$ is the vapor pressure. 


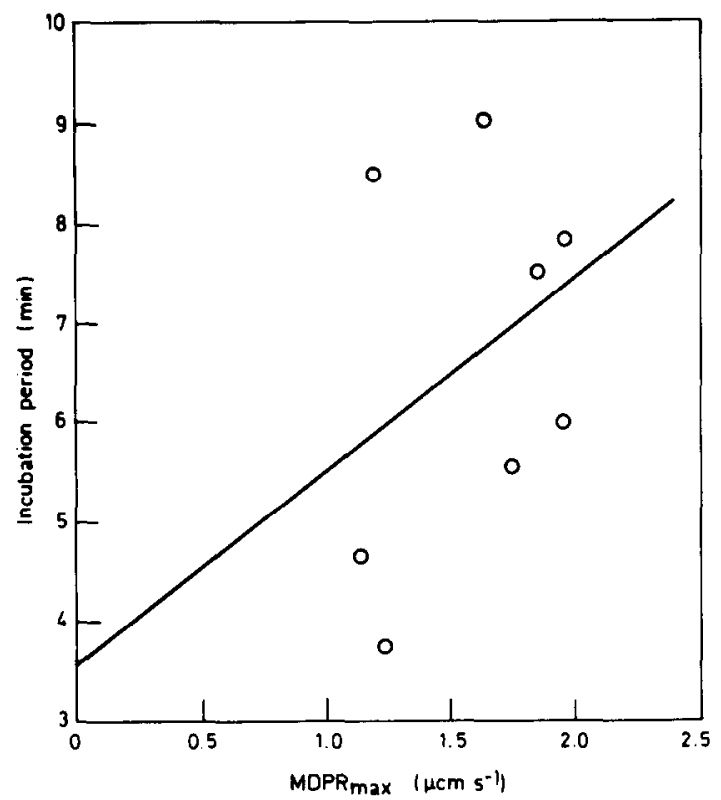

Fig. 5. Incubation period us. MDPR $\max _{\max }$ for stainless steel 304 in the vibratory facility: slope, $1.897 ; y$ intercept, 3.597 , correlation coefficient, 0.352 .

tude for these tests was 1.5 mil $(38 \mu \mathrm{m})$ and the horn frequency was $20 \mathrm{kHz}$ in all cases. MDPR $R_{\max }$ and the incubation period* were measured for each condition. All raw data and more complete test details are reported elsewhere [10].

Figures 3 and 4 show the relation between the ratio of MDPR $R_{\max }$ for freshwater tests to that for saltwater tests and MDPR $R_{\max }$ itself for 304 stainless steel and 1018 carbon steel respectively. For simplicity and reproducibility, the calculated least mean square best fit lines are shown rather than the best curves. The ratio increases toward unity for increased MDPR for all three materials, which indicates an increased mechanical intensity for these tests. The trend was greatest for carbon steel (Fig. 4) as might be expected and least for aluminum, with 304 stainless steel (Fig. 3) showing intermediate results.

Figure 5 shows the relation between incubation period and MDPR $R_{\max }$ for 304 stainless steel. The best least mean square fit straight line is again computed and shown. It would be expected on intuitive grounds plus much previous test experience that an increased MDPR $R_{\max }$ would correspond to a reduced incubation period, and both would correspond in general to an increased cavitation intensity for a given material or to weaker materials for a

*The incubation period is here defined as the intercept on the abscissa of a curve of weight loss versus test duration of the tangent drawn from the maximum slope portion of the curve. The incubation period is generally accepted as at least the second most important parameter (compared with $\mathrm{MDPR}_{\max }$ ) in quantifying cavitation resistance. 


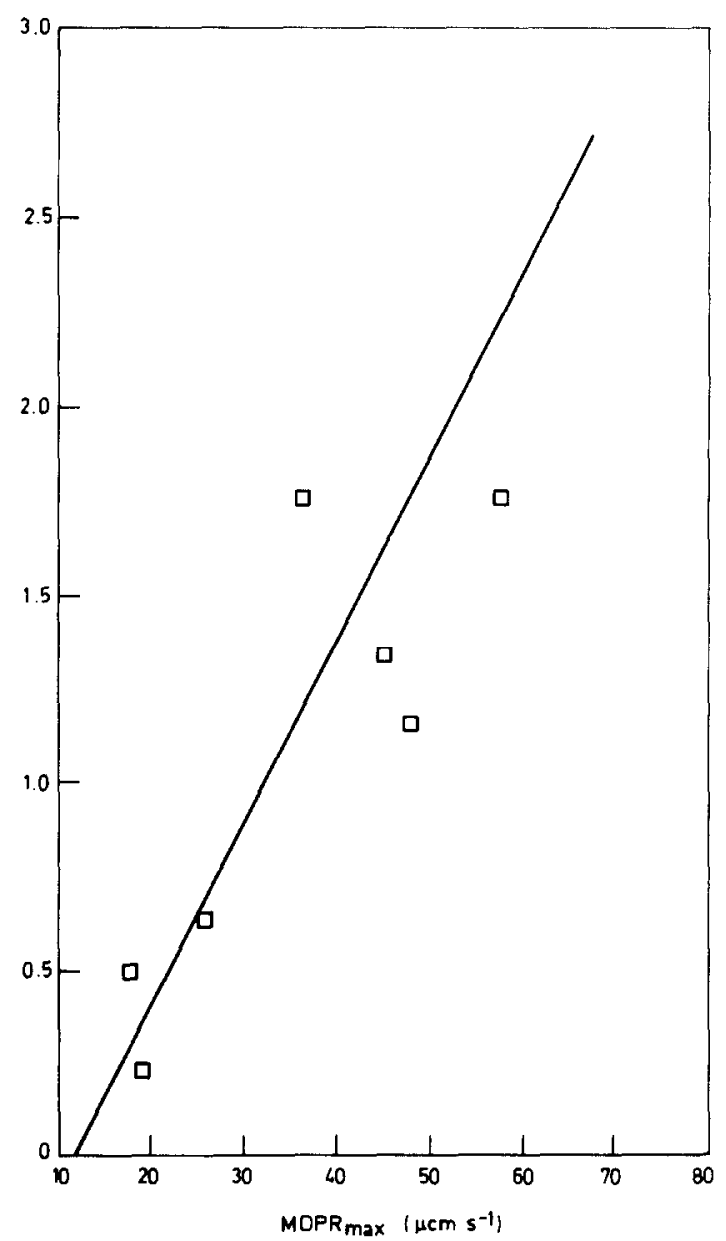

Fig. 6. Incubation period us. MDPR $\max$ for aluminum 1100-0 in the vibratory facility: slope, $0.0462 ; y$ intercept, -0.519 ; correlation coefficient, 0.883 .

given intensity. However, the inverse trend is shown in Fig. 5 for 304 stainless steel and is even more pronounced for 1100-0 aluminum (Fig. 6). The curve for carbon steel, which is not shown, is similar to that for 304 stainless steel [10]. However, the correlation coefficients are low and so these trends may not be meaningful.

Figure 7 combines the data for all materials in terms of the incubation period versus $\mathrm{MDPR}_{\max }$. In general the expected overall trend of decreasing incubation period for increasing $M D P R_{\max }$ is shown, but there is an unexpected intermediate minimum when results for all materials are combined on the same plot. This is caused by the strong inverse relation for aluminum as compared with the other materials. These results all indicate that the relation between the incubation period and MDPR $R_{\max }$ differs substantially according to the material tested. 


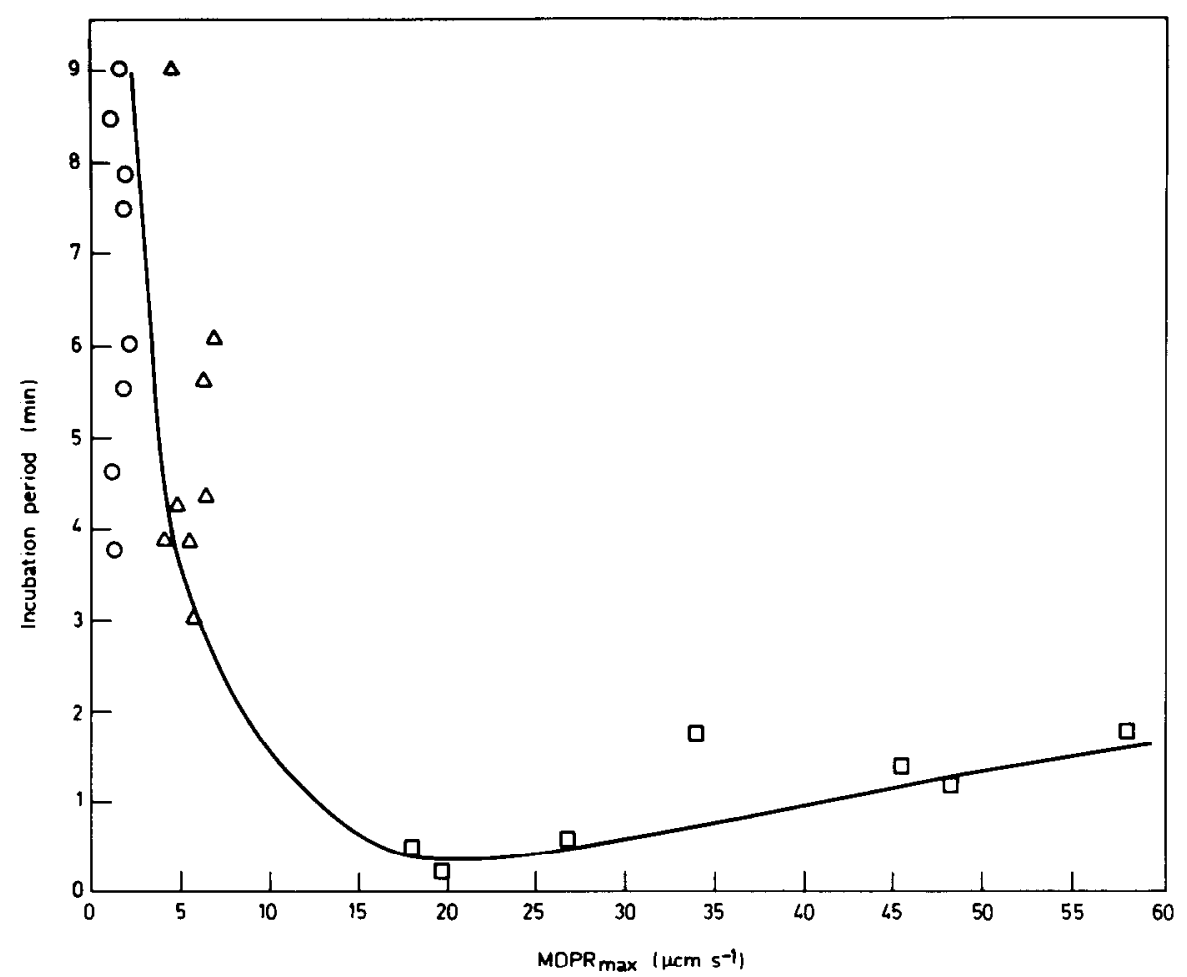

Fig. 7. Incubation period $v s$. $\mathrm{MDPR}_{\max }$ for all materials in vibratory facility: $O$ stainless steel $304 ; \triangle$ carbon steel $1018 ; \square$ aluminum $1100-0$.

\section{Venturi pressure pulses and damage [11]}

\subsection{Apparatus and procedures}

The venturi tests were carried out in the high speed water loop [12] which is capable of a throat velocity of up to about $65 \mathrm{~m} \mathrm{~s}^{-1}$. Figure 8 shows a schematic diagram of this facility. Although there are no valves in the loop, any desired cavitation condition and throat velocity can be attained through adjustment of pump speed and the cover gas pressure in the surge tank, which is connected to the low pressure tank. Some degree of deaereation (to about $30 \% \mathrm{STP}$ ) is possible through a bypass stream into a vacuum spray tank. The air content is measured by a Van Slyke apparatus. Any desired degree of cavitation in the test venturi can be obtained by a suitable adjustment of surge tank pressure and pump speed. A cooler is installed in the downstream tank to allow some control and adjustment of liquid temperature by balance between the work input to the pump and heat loss from the cooler.

Figure 9 shows the Plexiglass venturi used for the present test series, including the positions of the acoustic microprobes, the damage specimens and 


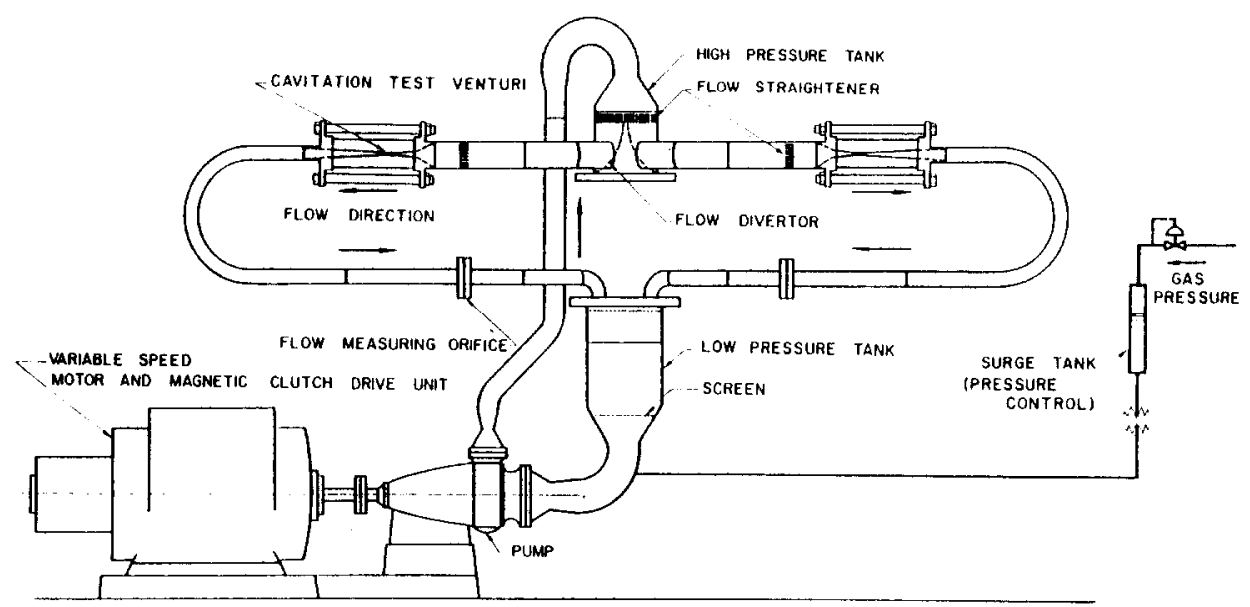

Fig. 8. Schematic diagram of the water cavitation facility (only two of the four loops are shown).

Cylindrical Venturi

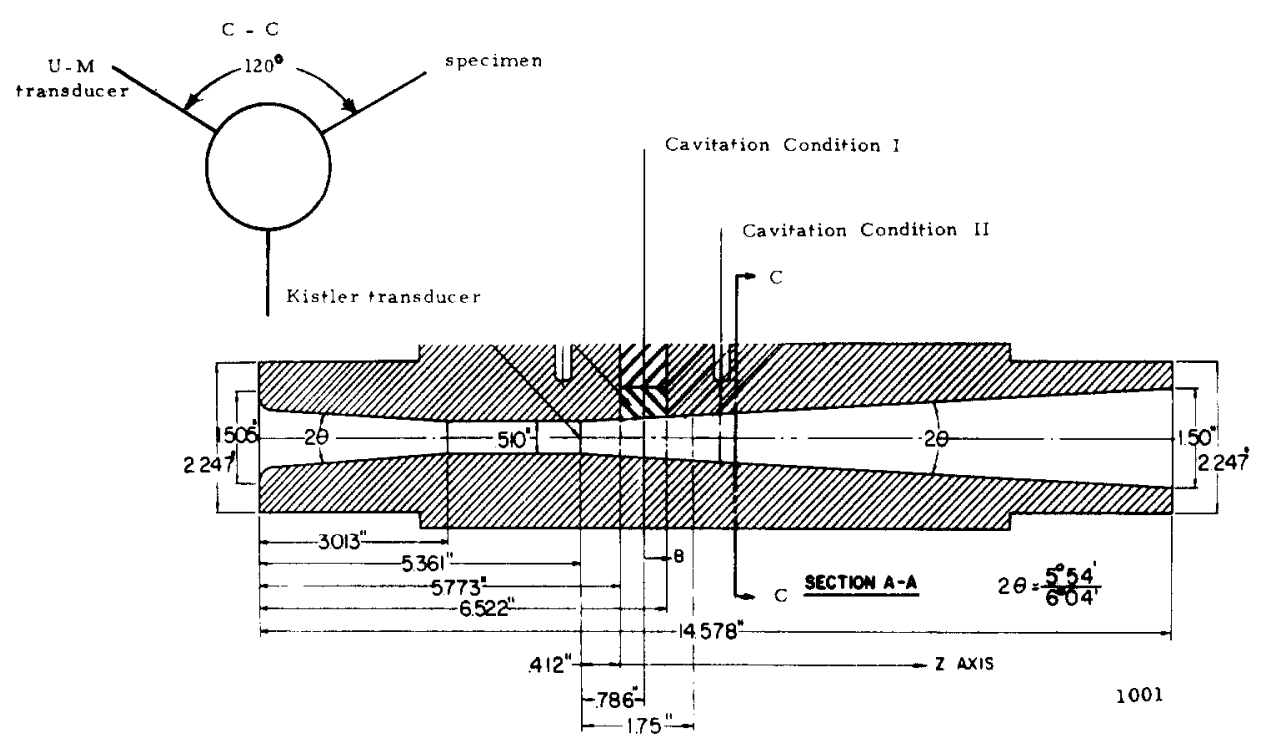

Fig. 9. Damage test venturi.

the extent of the cavitating region for cavitation conditions I and II. The venturi had been used for previous tests of a somewhat different type, and hence additional features, which are not pertinent to the present work, are included in this apparatus. As indicated in Fig. 9, the erosion sample and the two microprobes are located in the same plane, which is normal to the venturi axis at a point downstream of the throat exit. Thus, if axial symmetry is assumed, the erosion specimen (located flush with the venturi diffuser wall) sees the same cavitation field as the two microprobes, which are 


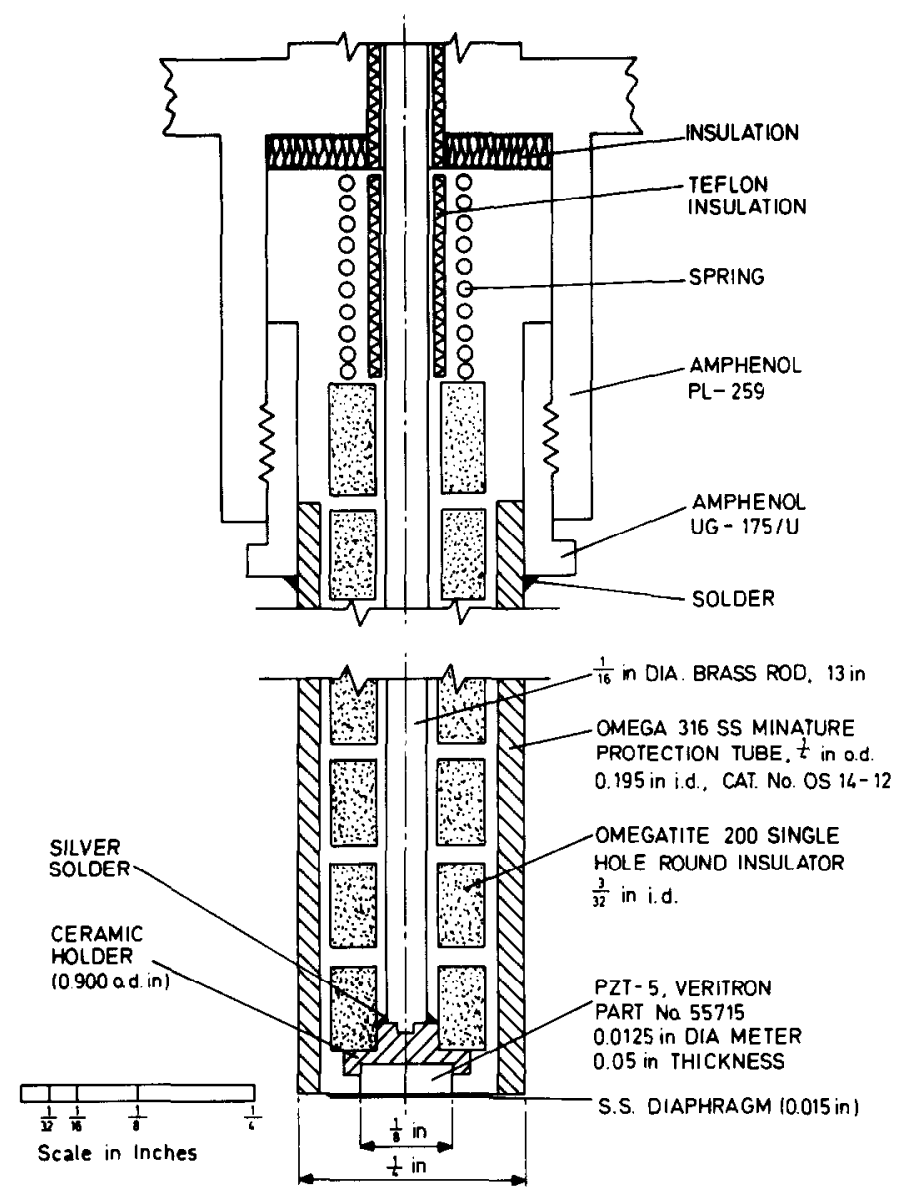

Fig. 10. University of Michigan high temperature acoustic probe.

also flush with the wall. Thus a direct correspondence is obtained between the pressure pulses measured by the two microprobes and erosion and pitting at an identical flow field location.

For cavitation condition II, the apparent end of the cavitating region is the plane of the probes and erosion specimens (1100-0 aluminum for the present tests). For cavitation condition I, the visually apparent cavitation ends $35 \mathrm{~mm}$ upstream of the probe position (Fig. 9). No doubt some more energetic and larger bubbles penetrate further.

Figure 10 shows the pressure microprobe designed and developed (U-M probe) for use in liquids (such as liquid sodium) at temperatures up to about $600{ }^{\circ} \mathrm{C}$. High temperature sodium tests were performed in the vibratory facility $[8,9]$, although such temperature capabilities were not required for the present tests. However, the necessity for high temperature performance with this probe did limit its natural frequency to about $0.1 \mathrm{MHz}$. It was calibrated against a commercial Kistler probe (Model 601A) which also proved to have a natural frequency of about the same magnitude. Both probes were used in 


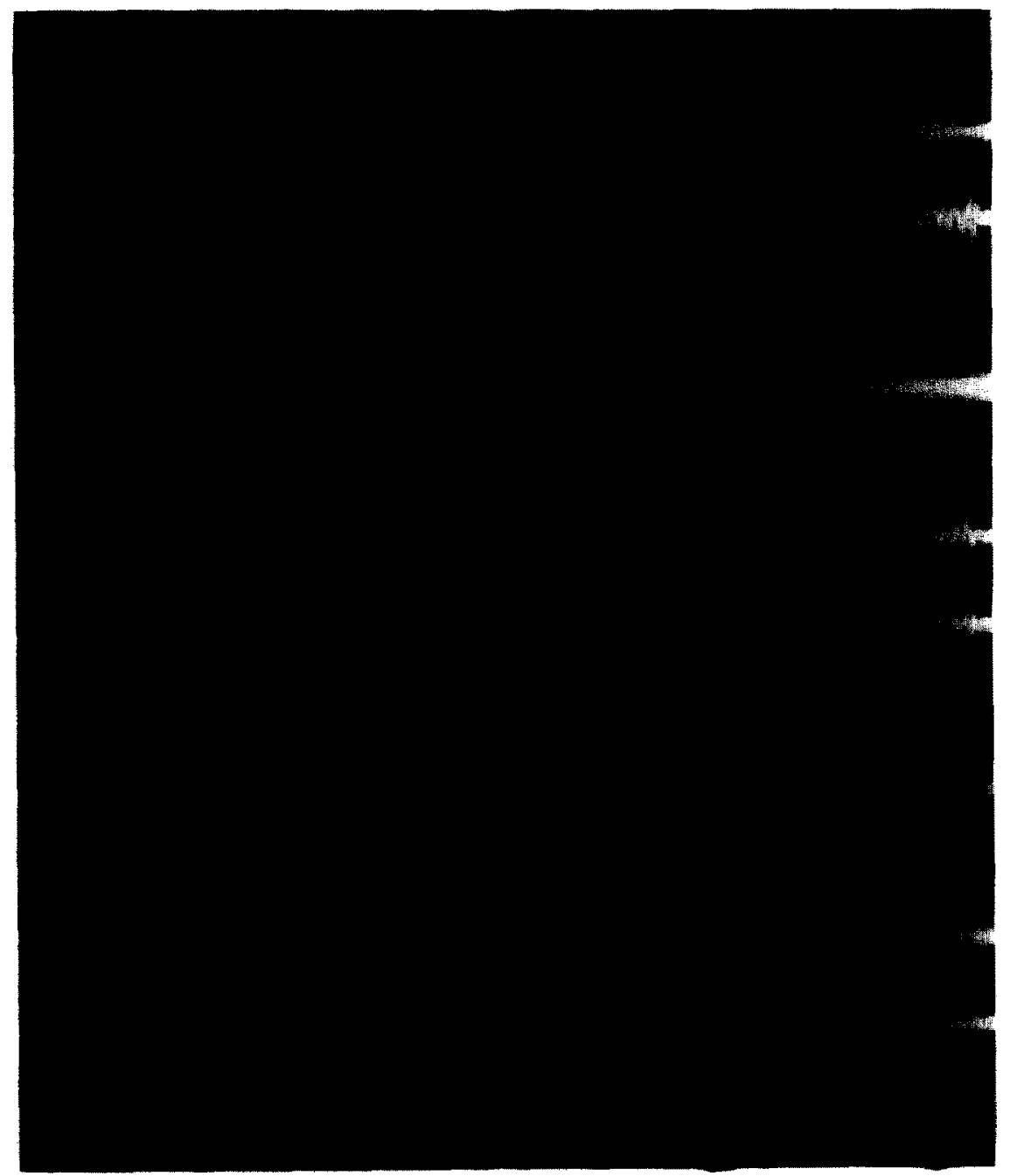

Fig. 11. Response of U-M and Kistler microprobes (positioned on a plane of symmetry in a venturi) to pressure waves emanated by collapsing cavities.

the present work to investigate the degree of axial symmetry of the cavitation field. These probes represent the approximate present state of the art in this field.

Ideally, the pulse height spectra for the various system conditions should be obtained via a multichannel analyzer (MCA). However, the complexity of the actual signal (Fig. 11) makes this approach difficult. On theoretical and experimental grounds [13] it is most likely that in many cases the rise time of the pressure pulses seen by the probes is considerably less than $1 \mu \mathrm{s}$, perhaps as little as $20 \mathrm{~ns}$ [13]. Since the period of the resonant vibration of each probe is about $10 \mu \mathrm{s}$, it is clear that they* cannot faithfully

*Or any presently available pressure probes. 
follow the pressure pulsations generated by the collapsing bubbles. Thus a single nearby collapse will generate a probe signal rise characteristic of its own natural frequency, no doubt seriously truncating the actual pulse amplitude. In addition, the initial impulse on the probe will be followed by probe "ringing" as seen clearly in Fig. 11 where the simultaneous outputs of both probes are displayed and seen to be quite similar. The small background noise in the absence of large pulses is probably primarily due to fluid turbulence and other noises of electronic origin.

It is apparent from Fig. 11 that a simple MCA counting circuit would register many collapses for each actual collapse owing to probe ringing. Thus a pulse-shaping circuit would be required for an eventual automatic device of this type for measuring cavitation mechanical intensity. In this preliminary work it was preferred to postpone this approach in favor of a more "manual" one capable of providing basic information on the actual signals obtained, and hence on the actual features of the bubble collapse, before launching into the design of a more automated MCA-type circuit. An alternative overall approach would be the development of microprobes of higher natural frequency, and this is now being investigated.

Pulse height spectra for the present study were developed by means of oscilloscope photographs and a threshold triggering circuit. Low frequency input, not of interest for cavitation bubble collapse, was suppressed by a high pass filter set at a frequency of about $90 \mathrm{kHz}$. Numerous oscilloscope photographs were taken for each of the different system parameters investigated because of the very large variations in pulse count at different amplitudes: $10^{6}-10^{7} \mathrm{~min}^{-1}$ for low amplitudes in comparison with $10 \mathrm{~min}^{-1}$ for large amplitudes. From the viewpoint of cavitation damage only the large amplitude pulses are of interest. This is confirmed by the already well-known observation from studies using high speed cinematography that only 1 in about $10^{4}-10^{6}$ bubbles appearing to collapse near the surface produces a visible crater even in soft materials $[14,15]$. Simple numerical calculations based upon previous motion pictures from our venturi [14] indicates that the number of bubbles traversing the venturi is such that on average they should be well separated, so that in general there should be little acoustic interference between different bubble collapses.

\subsection{Experimental results and discussion [11]}

\subsubsection{Pulse details}

Figure 11 shows the oscilloscope output from the Kistler and U-M probes for cavitation condition I and a downstream pressure of 1 bar (gauge) for water at a temperature of about $20^{\circ} \mathrm{C}$ so that vapor pressure is negligible. The throat velocity for this condition (Table 1 ) is about $12 \mathrm{~m} \mathrm{~s}^{-1}$. It is assumed that each of the individual pulse groups is due to the collapse of single bubbles. In those cases where each probe registered approximately identical outputs it is presumed that the bubble collapse occurred approximately equidistant from the two probes; i.e. not adjacent to either. This is the case for the first collapse in the upper right-hand photograph in Fig. 11. The second 
TABLE 1

Summary of venturi test parameters

\begin{tabular}{ccc}
$\begin{array}{l}P_{\text {out }} \\
\text { lbf in }^{-2} \text { gauge) }\end{array}$ & $\begin{array}{l}V_{\mathrm{I}} \mathrm{m} \mathrm{s}^{-1} \\
\mathrm{~m}^{-2}\end{array}$ & $\begin{array}{l}V_{\text {II }}-1 \\
\mathrm{~m} \mathrm{~s}^{-1}\end{array}$ \\
\hline 0 & 11.6 & 14.7 \\
10 & 13.7 & 16.8 \\
50 & 18.9 & 22.9 \\
50 & 28.9 & 36.6 \\
\hline
\end{tabular}

$P_{\text {out }}$, outlet pressure from venturi; $V_{\mathrm{I}}$, venturi throat velocity at cavitation condition I; $V_{\mathrm{II}}$, venturi throat velocity at cavitation condition II $\left(1 \mathrm{~atm}=14.7 \mathrm{lbf} \mathrm{in}^{-2}\right)$.

collapse seen by the Kistler probe in that case was apparently not seen by the U-M probe, and thus presumably took place close to the Kistler probe. However, a detailed examination of many such photographs indicated no systematic difference between the two probe positions, which confirmed the essential axial symmetry of the flow. Also, as observed in the upper right. hand photograph and elsewhere in Fig. 11, the first peak in each group is often not the highest. This may indicate that the registered amplitude is often severely truncated by the time response of the transducers. Further examination of Fig. 11 indicates the validity of the assumption that only single bubbles are in general active at a given time, which explains the relatively large time-axis spacings between the pulse groups.

Figure 12 shows a direct comparison of the pulse count spectra generated by the Kistler and U-M probes exposed to the same cavitation field. While there is some slight difference between the spectra, it is not great. In this particular case the U-M probe "sees" a somewhat greater number of collapses than does the Kistler probe.

\subsubsection{Pulse count spectra}

3.2.2.1. General. Figure 12 shows typical pulse count spectra generated from each probe in terms of counts per minute versus peak pressure (in pounds force per square inch). As already explained, the counts are the number of bubble collapses registered by the transducer. Pulse groups such as those shown in Fig. 11 are considered to correspond to a single collapse and thus form a single count.

In Fig. 13 pulse count spectra in terms of counts per minute versus peak pressure (maximum observed amplitude from transducer output) are compared for various conditions to investigate the effects of throat velocity (downstream tank pressure), the extent of the cavitating regime (cavitation conditions I or II, see Fig. 9) and the gas content. The preliminary results presented here show the interdependence of the pulse count spectra on all these parameters. 


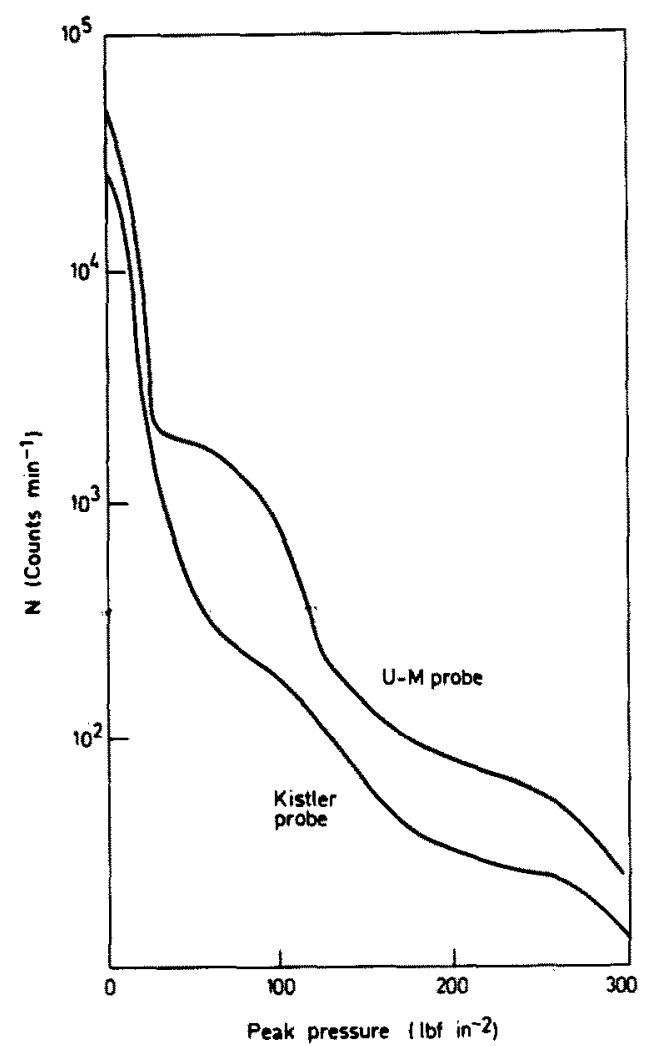

Fig. 12. Comparison of U-M and Kistler probes: integral distributions of pressure pulses (surge tank pressure, 1 atm gauge; cavitation condition II).

3.2.2.2. Effect of gas content. The gas (primarily air) content for these experiments, which was measured by a conventional Van Slyke apparatus, was varied between a maximum of slightly more than saturation at STP (i.e. about 2.0 vol.\%) to a fraction of that value (about $0.6 \%$ saturation at STP, i.e. about 1.2 vol.\%). For most of the runs the lower gas content was used, producing the upper two curves in Fig. 13. High total gas content (about 2.0 vol.\%) somewhat in excess of saturation (STP) was used for the lower three runs depicted (Fig. 13). A direct comparison is possible between the upper condition II curve for a back-pressure of $25 \mathrm{lbf}^{-2}$ (gauge) and the lower condition II curves for 1 and 6 bar (absolute). It is apparent that the number of counts producing any given peak pressure on the transducer was reduced by a factor of about $10^{2}$ compared with the runs at higher air content. If it is assumed that the spectral areas (areas under the $N$ versus $P$ curves) are roughly proportional to the count number at a given peak pressure and that the damage rate is roughly proportional to the spectral area $[8,9]$, then the damage rate (MDPR) would be reduced by about $10^{2}$ by such a change in gas content. No definite conclusions' of this type can yet be 


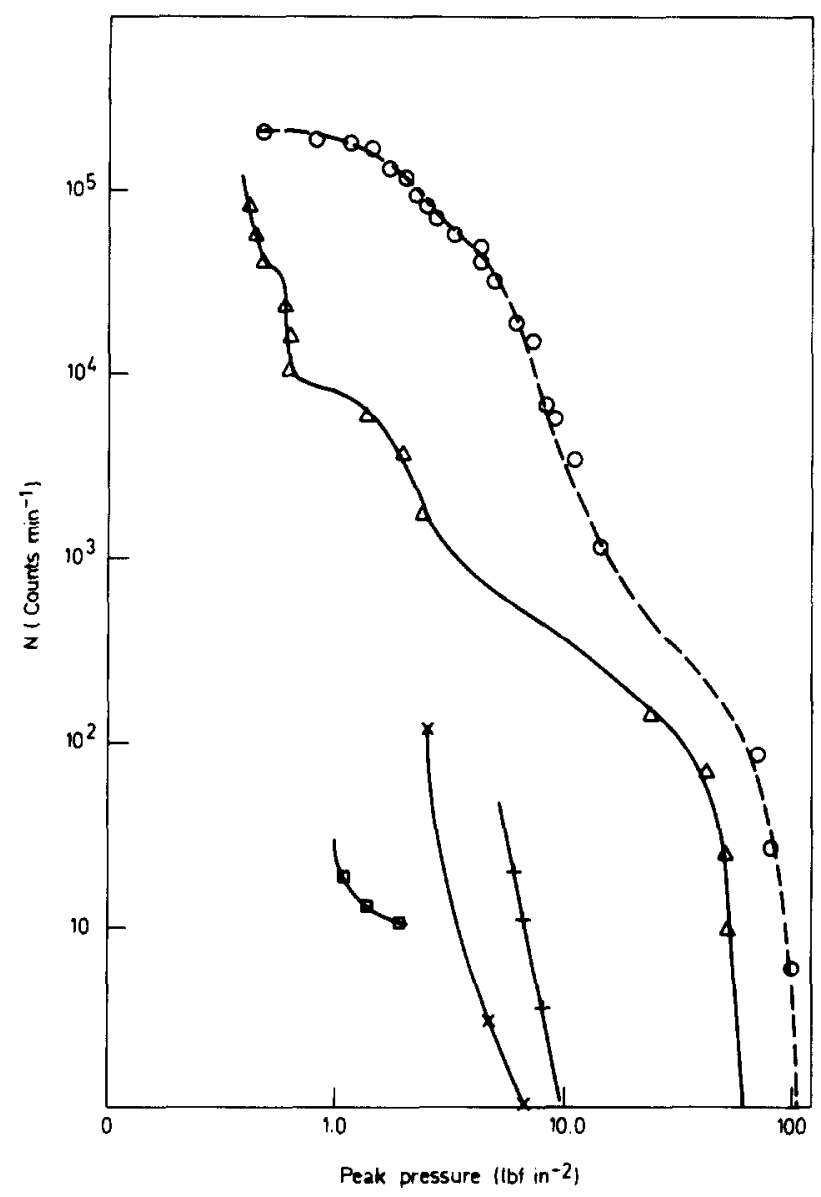

Fig. 13. Integral distributions of pressure pulses (U-M probe) for various flow conditions: $\circ, P_{\text {out }}=25$, cavitation condition II; $\triangle, P_{\text {out }}=50$, cavitation condition $\mathrm{I} ; \times, P_{\text {out }}=75$, cavitation condition II;,$+ P_{\text {oyt }}=150$, cavitation condition II; $\square, P_{\text {out }}=0$, cavitation condition II (pressures in lbf in ${ }^{-2}$ gauge).

drawn from the present work, since no quantitative erosion measurements have as yet been made. However, it has long been known [15 - 17] that an increase of air content in this range does significantly reduce MDPR.

3.2.2.3. Throat velocity and downstream pressure. The throat velocity increases with downstream pressure for a given cavitation condition (as shown in Table 1) in this venturi facility, and the expected strong increase of MDPR with velocity is well known [15-18]. This effect is also strongly illustrated in Fig. 13 for the three runs at high air content in the lower portion of the curve sheet. However, the effect is opposite for the two runs at low air content at cavitation condition $I$ in the upper portion of the curve. Since no quantitative damage data have been obtained in these tests, no final conclusions can be drawn. However, only a small damage effect due to velocity was observed in previous tests [14] with the same venturi geometry. 
3.2.2.4. Effects of cavitation condition. The effect of the cavitation condition, i.e. the extent of the cavitating region (Fig. 9), for fixed downstream pressure, i.e. approximately fixed velocity (Table 1), is shown in Fig. 13 by comparing two of the upper curves (back-pressure, $25 \mathrm{lbf}^{-2}$ (gauge)). The pulse count at large peak transducer pressures (important for damage) are greater by about a factor of 10 for condition II. Thus the more fully developed cavitation condition II produces a higher pulse spectrum than the cavitation condition closer to inception, i.e. condition I. This is partially because the probes are also located at position II (Fig. 9). Thus presumably less damage would be caused to probes located at position II by condition I than by condition II. This result occurs in spite of the fact that the bubble collapsing pressure at probe position II is greater for the less developed cavitation. Even though the cavitation region observed with the unaided eye in condition I does not extend to the probe position, presumably a sufficient number of large and energetic bubbles do penetrate that far. This conclusion is consistent with previous damage tests carried out in this venturi $[14,15]$.

3.2.2.5. Probe and actual surface peak pressure amplitudes [11]. The peak pressure amplitudes seen by the microprobes (e.g. Fig. 13, see ref. 11 for full details and other examples) extend up to about $10^{2} \mathrm{lbf} \mathrm{in}^{-2}$, which is obviously much too small to be damaging even to the $1100-0$ aluminum specimens. However, considerable damage was obtained (Fig. 14) for many of the conditions tested in runs of only 15 min duration. It is thus obvious that the measured pressure amplitudes are much smaller than those actually existing on the surface. In our opinion there are two major reasons for this discrepancy. The first and most obvious is geometrical. The active area of the probes (about $5 \mathrm{~mm}$ diameter) is much greater than the presumed microjet or shock-wave diameter from a bubble collapsing on and/or rebounding adjacent to the damaged surface [19]. In a previous study by Kling [19] using $10^{6} \mathrm{~Hz}$ motion pictures the jet diameter appeared to be about $0.1 \mathrm{~mm}$ from an initial bubble diameter of about $3 \mathrm{~mm}$. Thus the jet (or impinging shock) area is probably about a factor of $10^{2}-10^{4}$ greater than the probe area, giving a possible pressure multiplying factor of this order of magnitude on the basis of geometry. A second reason for the discrepancy between the measured and actual surface pressures is the inefficiency of the probes themselves in recording possible nanosecond rise times [13]* since the natural frequencies of the probe are about $0.1 \mathrm{MHz}$.

However, considering all uncertainties, peak surface pressures of about $10^{5}-10^{7} \mathrm{lbf}^{-2}$ are possible. The observed damage to very hard materials makes this range credible. While the present probes are obviously not ideal for measuring the actual peak surface pressure amplitudes for bubble collapse, the results should at least be proportional to the true values. Thus the pulse-

*Pressure probes with nanosecond rise times do not exist at this time. 


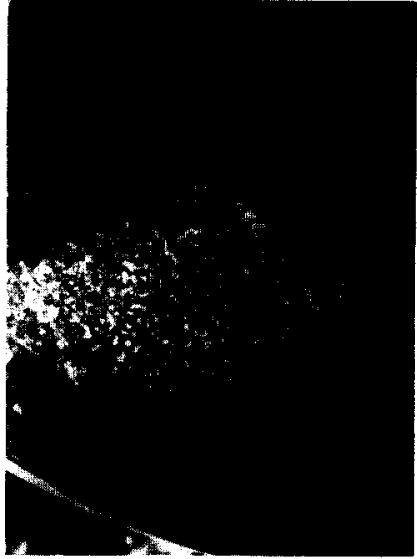

(a)

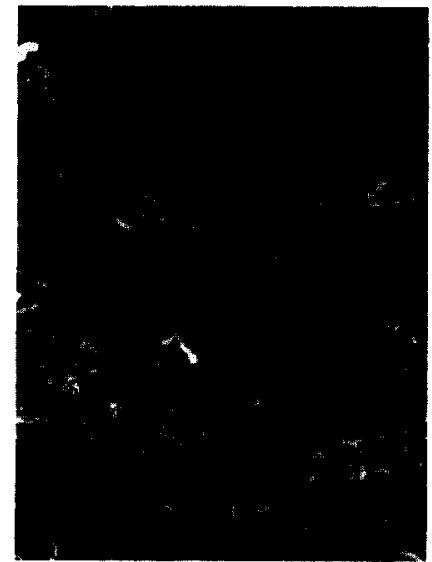

(b)

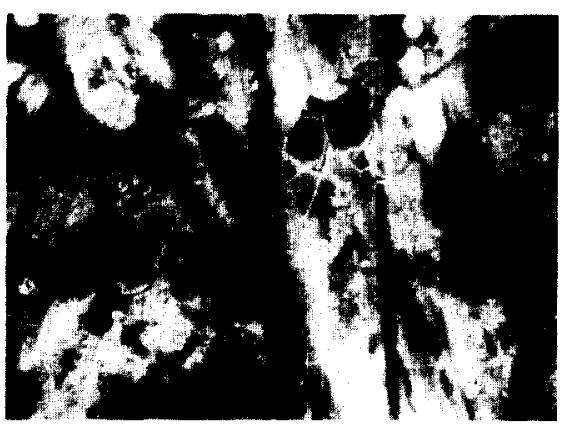

(c)

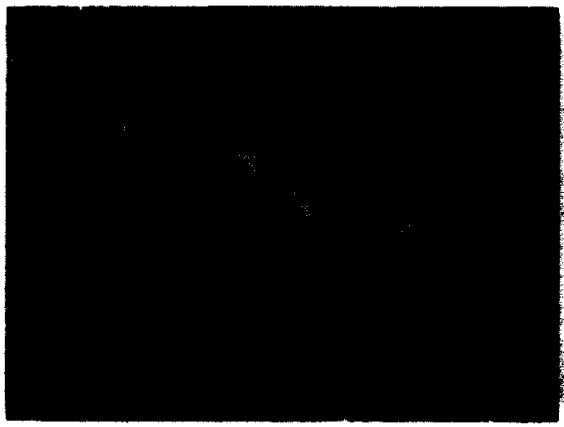

(d)

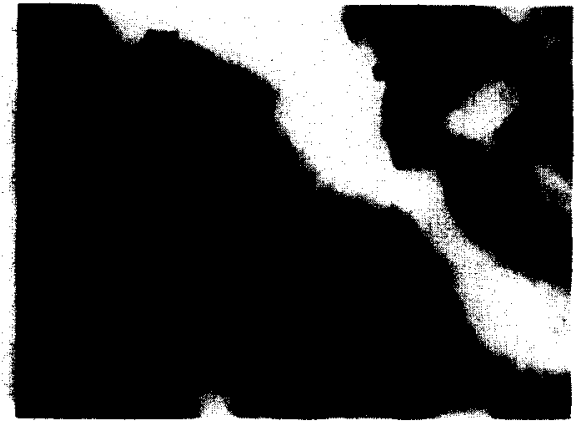

(e)

Fig. 14. Scanning electron micrographs of $1100-0$ deformed specimens: (a) $50 \times$; (b) $100 x$; (c) $250 x$; (d) $500 x$; (e) $1000 x$.

count spectra can be used for damage prediction once they are calibrated in an actual cavitation flow. We expect this to be accomplished when actual MDPR and pit-count data become available.

\subsubsection{Pulse count spectra and erosion}

Figure 15 shows the pulse count spectra developed for six different system conditions taken on the same day using the same loop water to avoid 


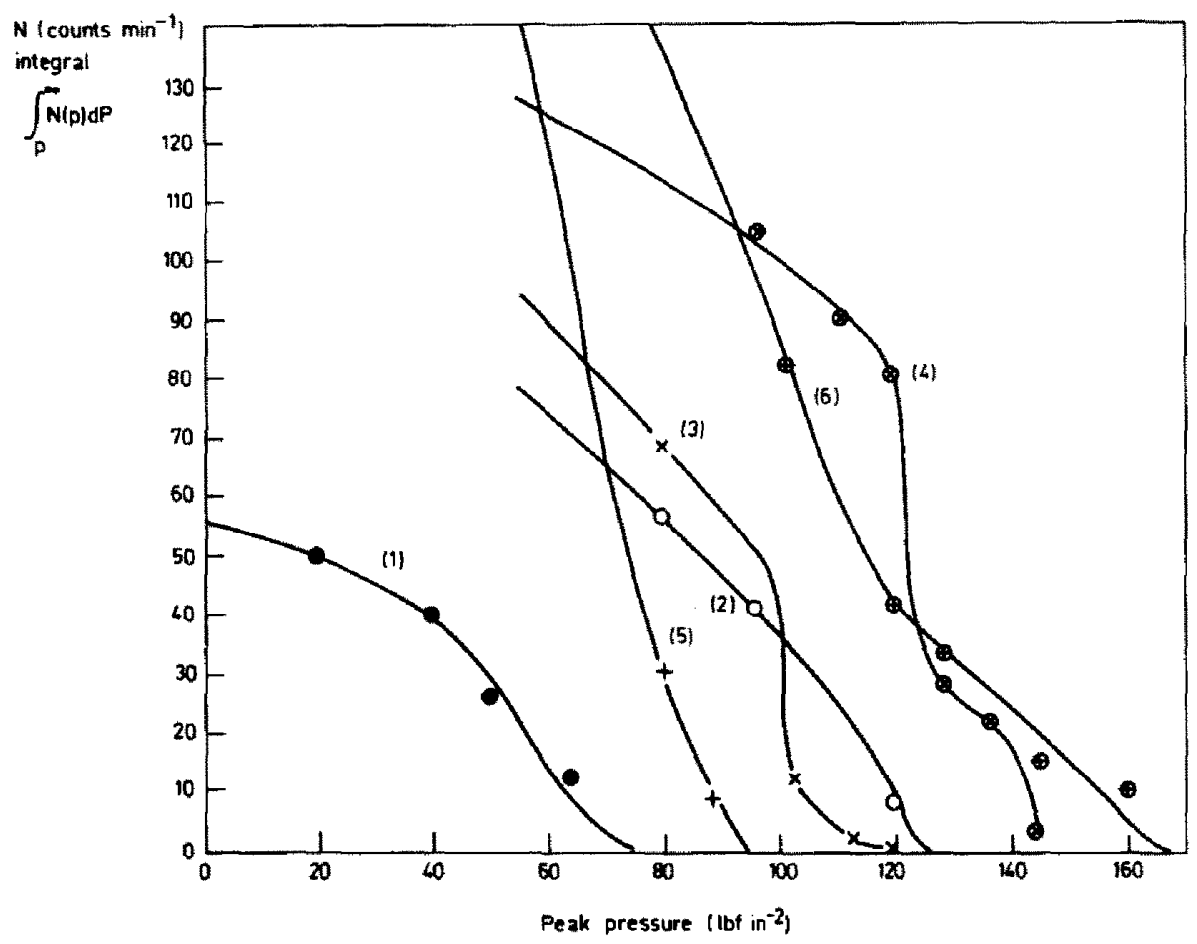

Fig. 15. Spectral distribution of pressure pulses for erosion tests for the following system pressures (lbf in ${ }^{-2}$ gauge) and cavitation conditions: curve 1, 50 , condition 1 ; curve 2 , 50 , condition II; curve 3,25 , condition I; curve 4,25 , condition II; curve 5,10 , condition I; curve 6,10 , condition II.

any variations. At the same time 1100-0 aluminum damage specimens of diameter 0.250 in $(6.4 \mathrm{~mm})$ were inserted flush with the wall at the same axial position as that of the microprobes (Fig. 9) and exposed to cavitation for $15 \mathrm{~min}$ in each case, during which time the pressure pulse spectra were generated. Thus in this condition there is a one-to-one correspondence between the pulse spectra and the damage observed. Figure 16 is a schematic representation of the damage obtained, and Fig. 14 shows scanning electron micrographs of the damaged specimens. No exact pit counts or weight loss measurements were made for these initial damage tests. However, it is apparent that significant damage was obtained only on specimens nos. $4-6$, and that these correspond to spectra showing appreciable numbers of counts in the region of higher peak pressure. This is particularly true of nos. 4 and 6 , while no. 5 is somewhat more marginal. A close examination of no. 5 shows less damage than for nos. 4 and 6 . Thus at least a qualitative correlation between the pulse count spectra and the observed damage was obtained for these preliminary tests. More precise damage tests are planned for the near future. 

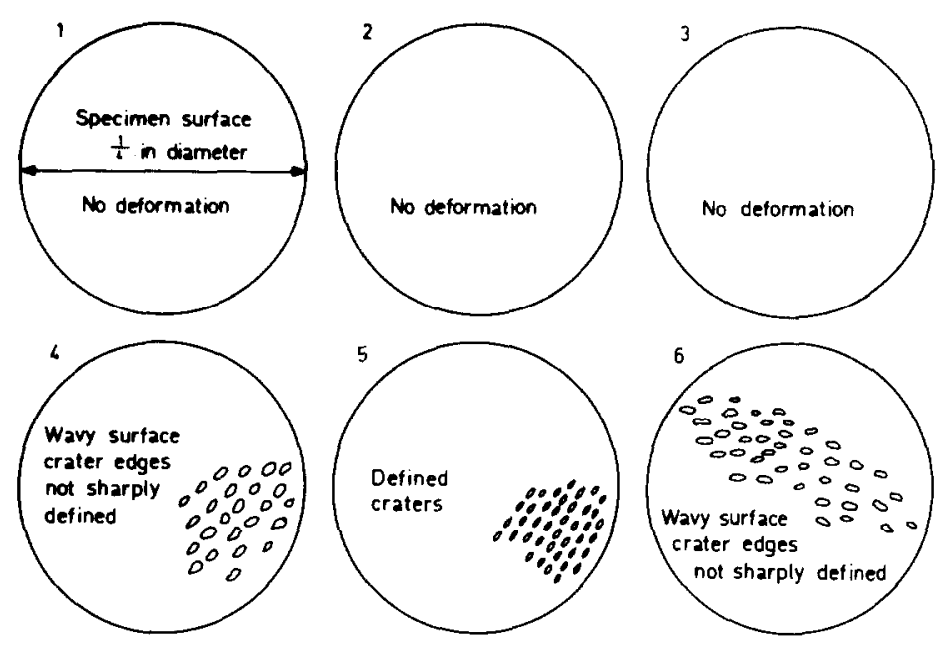

Fig. 16. Schematic diagram of $1100-0$ aluminum specimens after a 15 min exposure to cavitation. Specimen numbers correspond to curve numbers in Fig. 15.

\section{Conclusions}

(1) Cavitation damage tests carried out in a vibratory facility in synthetic seawater and tapwater, over a range of temperature and pressure conditions, with three materials of varying degrees of corrodability indicate that the ratio between the maximum MDPRs for saltwater and freshwater tests increases toward unity as the mechanical cavitation intensity is increased toward the maximum damage condition either by increasing the suppression pressure or varying the liquid temperature. This result is expected on theoretical grounds.

(2) As expected the incubation period increases for reduced maximum MDPR in the vibratory facility tests if the results for all the materials listed, i.e. 304 stainless steel, 1018 carbon steel and 1100-0 aluminum, are taken together. However, it is clear that the precise nature of the relation between the incubation period and the maximum MDPR depends on the properties of the materials themselves.

(3) A technique involving the observation of pulse height spectra from submerged pressure microprobes has been developed and used in a cavitating water venturi to measure the mechanical intensity of the cavitation bubble collapse and to correlate this parameter with the observed erosion. Only a qualitative correlation has so far been obtained in the venturi, although more precise correlations were obtained previously using an earlier version of the same system in the vibratory facility $[8,9]$.

(4) Pulse height spectra have been used in the present study to measure the effect on the mechanical cavitation intensity of various parameters such as total gas content, throat velocity and cavitation condition (extent of 
cavitating region). The results are reasonably consistent with previous predictions.

(5) The microprobes used (one developed by us for high temperature use and one commercial probe) are suitable for providing a practical means of measuring mechanical cavitation intensity and thus providing an a priori prediction of the eventual erosion rates pertinent to various flowing laboratory and field devices. The resultant pulse height spectra can be used to measure a cavitation erosion efficiency which compares the acoustic power delivered to an eroded surface with erosion power, which is defined as the product of the ultimate resilience of the material and the volume loss rate $[8,9]$.

(6) The microprobes used are not capable of following in detail the pressure pulsations resulting from bubble collapse in a cavitating field owing to an insufficient time response rate. However, for probes of practical utility and adequate ruggedness, it may not be possible greatly to improve this situation. The present probes appear adequate for the purpose of damage prediction and represent the present state of the art in this regard.

\section{Acknowledgments}

This work was supported by the Office of Naval Research Contract No. N00014-76-C-0697 and by internal University of Michigan funds (SEP and Work Study). Mr. Stanley W. Doroff was the ONR project monitor.

\section{References}

1 I. S. Pearsall and P. J. MeNulty, Comparisons of cavitation noise with erosion, 1968 ASME Cavitation Forum, ASME, New York, 1968, pp. 6 - 7.

2 W. M. Deeprose, N. W. King, P. J. McNulty and I. S. Pearsall, Cavitation noise, flow noise and erosion, Proc. Conf. on Cavitation, Herriot-Watt Univ., Edinburgh, Sept. 1974, Inst. Mech. Eng., London, 1974, pp. $373-381$.

3 J. J. Varga and Gy. Sebestyen, Determination of hydrodynamic cavitation intensity by noise measurement, Proc. 2nd Int. JSME Symp. on Fluid Machinery and Fluidics, Tokyo, Sept. 1972, pp. $285 \cdot 292$.

4 J. J. Varga, Gy. Sebestyen and A. Fay, Detection of cavitation by acoustic and vibration-measurement methods, Houille Blanche, 2 (1969) 137 - 149.

5 F. Numachi, Transitional phenomena in ultrasonic shock waves emitted by cavitation on hydrofoils, J. Basic Eng., 81 (June 1959) 153.

6 F. Numachi, An experimental study of accelerated cavitation induced by ultrasonics, J. Basic Eng., 87 (1965) 967 - 976.

7 V. K. Mukarov, A. A. Kortnev, S. G. Suprun and G. I. Okolelov, Cavitation erosion spectra analysis of pulse-heights produced by cavitation buhbles, Proc. 6th NonLinear Acoustics Conf., Moscow, July 1975.

8 F. G. Hammitt, S. A. Barber, M. K. De and A. N. El Hasrouni, Predictive capability for cavitation damage from bubble collapse pulse count spectra, Proc. Conf. on Scaling for Performance Prediction in Rotodynamic Machines, Univ. of Stirling, Sept. 1977, Inst. Mech. Eng., London. 
9 F. G. Hammitt, S. A. Barber, M. K. De and A. N. El Hasrouni, Cavitation damage prediction from bubble collapse pulse count spectra, 1977 ASME Cavitation and Polyphase Flow Forum, June 1977, ASME, New York, 1977, 25 - 28.

10 F. G. Hammitt, Ph. N. Hasrouni, A. N. El Hasrouni and G. Vaghidas, Vibratory facility cavitation damage tests: tap water vs. synthetic sea water, ORA Rep. no. UMICH 014456-18-I, Univ. Michigan, Ann Arbor, Mich., May 1977.

11 M. K. De, Emission and transport of shock waves from pulsating cavities in high speed fluid flow and their incidence on a solid surface, ORA Rep. no. UMICH 014456-22-I, Univ. Michigan, Ann Arbor, Mich., July 1977.

12 F. G. Mammitt, Cavitation damage and performance research facilities, ASME Symp. on Cavitation Research Facilities and Techniques, 1964, ASME, New York, 1964, pp. $175 \cdot 184$.

13 W. Lauterborn and K. J. Ebeling, High-speed holography of laser-induced cavitation bubbles in liquids, Proc. 7th Int. Symp. on Nonlinear Acoustics, Aug. 1976, Blacksburg, Va.

14 M. J. Robinson and F. G. Hammitt, Detailed damage characteristics in a cavitation venturi, J. Basic Eng., 89 (1) (1967) $161-173$.

15 R. T. Knapp, J. W. Daily and F. G. Hammitt, Cavitation, McGraw-Hill, New York, 1970.

16 R. E. H. Rasmussen, Some experiments on cavitation erosion in water mixed with air, Proc. 1955 NPL Symp. on Cavitation in Hydrodynamics, Her Majesty's Stationery Office, London, 1956, Paper 20.

17 J. M. Mousson, Pitting resistance of metals under cavitation conditions, Trans. ASME, 59 (1937) 399 - 108.

18 R. T. Knapp, Recent investigations of cavitation and cavitation damage, Trans. ASME, 77 (1955) $1045-1054$.

19 C. L. Kling and F. G. Hammitt, A photographic study of spark-induced cavitation bubble collapse, J. Basic Eng., 94 (4) (1972) 825 - 833. 